\title{
The effects of preexposure to a nonattended stimulus on subsequent learning: Latent inhibition in adults
}

\author{
A. GINTON, G. URCA, and R. E. LUBOW \\ Tel-Aviv University, Ramat-Aviv, Israel
}

\begin{abstract}
An experiment was designed to demonstrate the latent inhibition phenomenon in adult humans and to determine the effects of initial level of attention to the to-be-conditioned stimulus during a preexposure phase on subsequent learning. Results indicate that a strong latent inhibition effect can be obtained with adult humans. This effect occurs even though the preexposed stimulus is presented under severe conditions of inattention.
\end{abstract}

The phenomenon of latent inhibition, reviewed by Lubow (1973a), is defined as the decremental effect of nonreinforced preexposure of the to-be-conditioned stimulus on subsequent learning. Although latent inhibition has been found to be a robust phenomenon occurring in a wide variety of species and learning tasks, one notable anomaly has been the difficulty in demonstrating it in adult humans. Fifty-nine of 62 experiments using either infrahumans or children report latent inhibition (Lubow, 1973a), while only 5 of 15 studies with adults report latent inhibition (Lubow, 1973b).

It would appear, then, that there is a significant phylogenetic and/or developmental factor which interacts with nonreinforced preexposure of the to-be-conditioned stimulus to produce latent inhibition in lower animals and children but to retard it in human adults. An analysis of the differences in procedure between those studies using adult human subjects which obtain latent inhibition and those that do not obtain it suggests that attentional processes may account for the differential successes in obtaining latent inhibition in humans and, therefore, may also account for the phylogenetic developmental anomaly.

It is suggested that the necessary condition for producing latent inhibition is the lack of an attentional response to the to-be-conditioned stimulus in the formal learning phase of the paradigm. One way of achieving the condition of inattention is through nonreinforced presentation of that stimulus during the preexposure phase. This procedure is sufficient for producing latent inhibition in infrahumans and children, but insufficient for adults. The normal conditions of nonreinforced preexposure must be very puzzling indeed for the adult subject. In an empty sound-attenuated chamber with only a tone sounding every $30 \mathrm{sec}$ or so, if anything, the subjects' expectation that something is going to happen with that tone may well increase over presentations

This research was supported, in part, by the Israel Trustees Foundation Grant 2958 to R. E. Lubow. Requests for reprints should be sent to R. E. Lubow, Department of Psychology, Tel-A viv University, Ramat-Aviv, Israel. rather than decrease! This expectancy effect could be obviated by constructing the preexposure phase of the experiment in a manner such that the to-be-conditioned stimulus is exposed within a masking situation which provides a plausible explanation for the presence of the stimulus.

Support for the above speculations is provided by the fact that the only study to demonstrate latent inhibition in adult humans using CS preexposure and eyelid conditioning (Schnur \& Ksir, 1969) is one which employed a masking task (Spence, 1966). Five others, without the masking task, failed to obtain latent inhibition (Allen, 1967; Grant, Hake, Riopelle, \& Kostlan, 1951; Grant, Hake, \& Schneider, 1948; Perlmuter, 1966; Schnur, 1967). The only other testing paradigm for latent inhibition with a sufficient number of studies to evaluate is that of GSR. The results are decidely mixed. Some find the latent inhibition effect (Sokolov \& Paramanova, 1963; Surwit \& Poser, 1974, Wolff \& Maltzmann, 1968), and some obtain either no difference in conditioning as a function of nonreinforced preexposure (Zeiner, 1970) or a facilitatory effect (Silver, 1973). Unfortunately, the procedures are sufficiently different in so many respects as to make any valid comparison impossible.

The absence of attention, then, to the CS at the beginning of the learning phase is considered to be the major requirement for producing the latent inhibition effect. This lack of attention may be regarded as an acquired inattention which is developed through the preexposure phase. Thus, the preexposure phase typically starts with the presence of an attentional response to the to-be-CS which diminishes as a function of the number of preexposures. However, what happens if the attentional response to the to-be-CS is not elicited at the beginning of the preexposure phase? If in the typical masking task, it is assumed that the subject is aware of the presence of the CS but learns to ignore it, what then is the result of having that same stimulus presented under extreme conditions of inattentiveness? To rephrase this question: Is an initial attentional response to the to-be-CS during the preexposure phase 
necessary to produce the latent inhibition effect during the learning phase?

In summary, then, the two major interests of this paper are (1) to demonstrate latent inhibition in adult humans, and (2) to assess the effect of initial attention level to the to-be-learned stimulus, during the preexposure phase, on subsequent learning. To accomplish these objectives, attention was manipulated during the preexposure phase with a dichotic listening task, by presenting the CS to either that ear which the subject was monitoring, but for other information, or to that ear that was not being monitored. The first group was considered to represent a condition of preexposure under relatively high attention as compared to the second group. Two other groups received no preexposure to the to-be-learned stimulus and are considered to have the highest level of attention at the beginning of the learning phase.

\section{METHOD}

\section{Subjects}

Forty undergraduate psychology students from Tel-Aviv University, average age 21 years, were randomly divided into four groups, with the restriction that two males and eight females be included in each group.

\section{Apparatus}

Two lists, each consisting of 40 two nonsense-syllable pairs, were recorded on two separate tracks of a TC-270 Sony stereophonic tape recorder. The two lists were identical in content with only the order of presentation reversed. The interval between the beginning of each pair of syllables and the beginning of the next pair was 1 sec. Each list was recorded a number of times on the same track so that there was no indication as to the termination or restart of the list. Both tracks were rerecorded, but this time the CS, a white noise of an average of $1.25 \mathrm{sec}$ duration $(.5-2 \mathrm{sec})$ was superimposed randomly only on one track, with the restriction that 4-6 CSs appear in each 40 pair list. Thus, one set of recordings contained only verbal material on both tracks, Recording A. The other set of recordings was identical to the first except that a CS appeared on one of the tracks, Recording B.

Stimulus material was presented to the subject through Ando SA-2, 8-ohm reactance stereo earphones. An additional set was connected in parallel to enable the experimenter to monitor the recording. The verbal material was presented at an average of $-15 \mathrm{~dB}$ and the $\mathrm{CS}$, when appearing, at $-30 \mathrm{~dB}(0 \mathrm{~dB} 1 \mathrm{mv}$ at $600 \mathrm{ohm})$

\section{Procedure}

The subjects were seated in a sound-attenuated room. The tape recorder was placed on a table in front of the subject. A $60 \times 70 \mathrm{~cm}$ mirror stood behind the table, so that the experimenter, who was seated beside the subject throughout the experiment, could monitor the subject's eye movements. This was done in order to have an objective procedure for determining whether the subject was following instructions and attending to the appropriate ear during the preexposure phase. The direction of eye movement is highly correlated with the ear attended to (Gopher, 1973).

Preexposure phase. The subjects in all groups were told to attend to one ear, to count the number of times the list of paired syllables repeated itself in that one attended-to ear, and to report, at the end of the preexposure phase, the number of repetitions. The ear to which the subjects were told to attend and the stimulus material presented to that ear differed according to the group to which the subject belonged.

The subjects were divided into four groups. Two of the groups were preexposed to List B which included the to-be-conditioned stimulus and two of the groups were preexposed to List A, which did not include the to-be-conditioned stimulus. Of the two CS preexposed groups, one group received the to-be-conditioned stimulus in the ear that was monitored for the nonsense syllable repetition (PE/M-preexposed-monitored group). The other CS preexposed group received the to-be-conditioned stimulus in that ear opposite to the monitored one (PE/NM-preexposed-nonmonitored group). Both of these groups were subsequently tested with the conditioned stimulus presented to the same ear in which it was preexposed. Of the two groups not preexposed to the to-be-conditioned stimulus, one group was tested with the critical stimulus presented to that ear which was monitored during the preexposure phase (NPE/M-nonpreexposed-monitored group). The other group was tested with the conditioned stimulus presented to that ear which was not monitored during the preexposure phase (NPE/NM-nonpreexposed-nonmonitored group).

For half the subjects in each group the monitored ear was the right one, and for half it was the left one. The subjects were exposed to five repetitions of the 40-item list. This resulted in 26 exposures to the CS in the preexposed group. The subjects were not told that a testing phase would follow the preexposure phase.

Testing phase. After termination of the preexposure phase, all subjects were given the following instructions: "We are now starting with a new test. In this test all the stimulus material that you will hear on any track may be relevant. During presentation of the recording you will see the experimenter raising points on a scoreboard; the raising of points is not arbitrary but is guided by a rule connected directly to the information we will be hearing on the recording. The rule is a $100 \%$ rule. Listen to the recording and watch the scores rise. The minute you think you have caught on to the rule push the button in front of you whenever you expect me to raise a point. If your decision was correct a point will be subtracted instead of raised on the scoreboard. If you have made a wrong decision an additional point will be raised on the scoreboard. Your aim is to try and bring your score down to zero. Do not stop when you have reached zero, but go on pressing. I will tell you when to stop."

The scoreboard, a $3 \times 7-\mathrm{cm}$ metal box with two light-emitting diodes, was placed in front of the subject in the center of his visual field. Scores were changed manually by the experimenter through a remote control switch.

All groups were then exposed to Recording B. A point was added on the scoreboard each time the CS appeard and the subject did not push the button. The experiment terminated either after the subject had pressed five consecutive times to the CS, or after 31 presentations of the CS, i.e., six cycles of the 40 stimulus pairs if correct responding had not begun. ${ }^{1}$

The criterion for learning was the number of CSs to termination of experiment, not including the five correct presses to the $\mathrm{CS}$ if the subject had solved the problem. After termination of the experiment all subjects in the stimulus preexposure groups were told what the CS was and were asked whether they had heard it during the preexposure phase.

\section{RESULTS}

The monitoring of eye movements during preexposure indicated that all subjects attended to the ear designated in the instructions.

It can be seen from Figure 1 that most of the subjects 


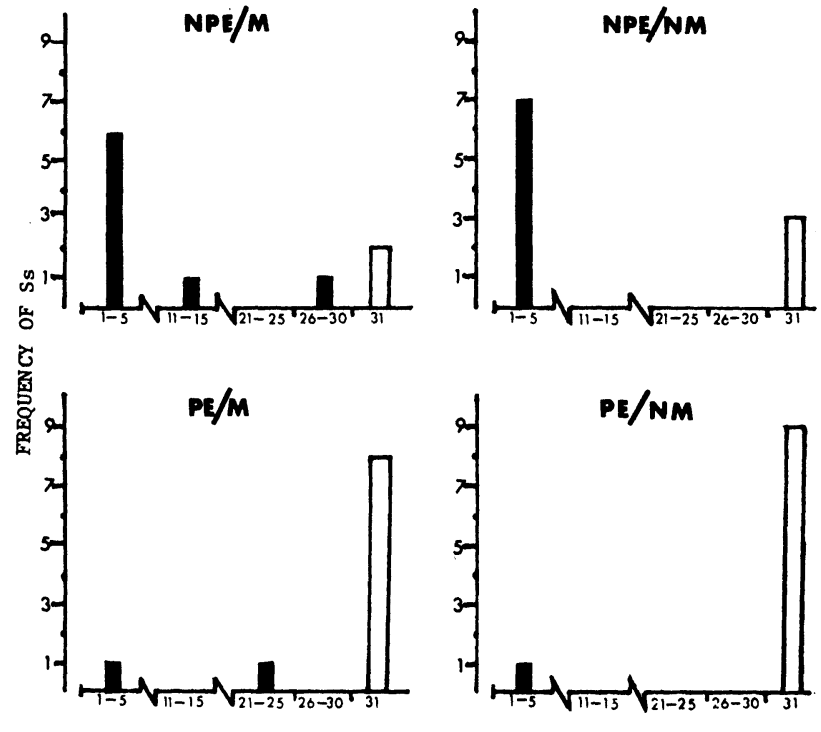

NUMBER OF CSS TO CRITERION

Figure 1. Distribution of subjects according to the number of CS presentations to reach the learning criterion. (Blank bars indicate failure to reach the criterion).

who solved the problem did so within the first few CS presentations. These subjects were concentrated in the groups which were not preexposed to the to-be-learned stimulus.

A comparison of the number of trials to reach the learning criterion for the subjects preexposed to the critical stimulus (Groups PE/M and PE/NM) as compared to groups not preexposed to the critical stimulus (Groups NPE/M and NPE/NM) yielded differences in favor of the nonpreexposed groups. This latent inhibition effect was highly reliable $(U=73.5, p<.001$, one-tailed).

The difference between the two $\mathrm{M}$ groups was found to be significant $(U=15, p<.01$, one-tailed $)$, as was the difference between the two NM groups $(U=17, p<.01$, one-tailed), thus indicating that there was a latent inhibition effect irrespective of whether preexposure of the to-be-conditioned stimulus was to the monitored ear or to the nonmonitored ear.

The two NPE groups were compared and were found not to be significantly different $(U=44, p>.10$, two-tailed). The difference between the two PE groups was also found not be significant $(U=46, p>.10$, two tailed).

Eight of the subjects in the $\mathrm{PE} / \mathrm{M}$ group reported hearing the CS during the PE phase, whereas only one subject in the PE/NM group reported hearing the CS during preexposure.

\section{DISCUSSION}

The experiment successfully demonstrated latent inhibition, as can be seen from the difference between the PE and NPE groups, but failed to demonstrate any weakening of the latent inhibition effect as a result of not attending to the to-be-conditioned stimulus during the preexposure phase (PE/NM group). One could question whether the procedure for the $\mathrm{PE} / \mathrm{NM}$ group was successful in eliminating attention. If attention is defined as "a process by which certain percepts attain access to the motor and long term memory systems" (Milner, 1970 , p. 294), then it would appear that the procedure was successful in eliminating the attention response in the $\mathrm{PE} / \mathrm{NM}$ group. This can be seen from the fact that only one out of 10 subjects in the $\mathrm{PE} / \mathrm{NM}$ group reported hearing the $\mathrm{CS}$ during the preexposure phase, whereas eight out of the 10 subjects in the PE/M group reported hearing the CS during preexposure.

However, even if the position is taken that attending to a stimulus does not necessarily imply remembering it, one would expect the experimental procedures to produce two different levels of attention to the to-be-CS. Why then, if attention is a crucial variable in latent inhibition, were there not differential effects of the two preexposure conditions on latent inhibition? The fact that no difference in latent inhibition was found in the two preexposure groups might not contradict the assumed criticality of attention for latent inhibition. It is possible that due to the number of preexposures to the CS, a ceiling effect had occurred and both groups had reached a maximal level of latent inhibition. This could be checked with additional groups, varying the number of preexposures.

In summary, this experiment clearly demonstrated latent inhibition in human adults. The procedure differed from other experiments with adult subjects (e.g., Schnur \& Ksir, 1969) in using an instrumental conditioning procedure, but similar to most other successful demonstrations of latent inhibition with adults, it used a masking task. It is suggested that masking reduces the stimulus' salience and thereby enhances the occurrence of inattention. In the present experiment the white noise CS, within a context of an experiment involving listening to recorded material, may be perceived by the subjects as part of the background noise and therefore result in inattention to that stimulus. What remains puzzling in this experiment is that having heard the CS and learned to disregard it has no greater inhibitory effect than having the CS fall on "deaf ears."

\section{REFERENCES}

Allen, J. A. Latent inhibition: Generalization during eyelid conditioning. Unpublished master's thesis, North Carolina State University, 1967.

Gopher, D. Eye movement patterns in selective listening tasks of focused attention. Perception \& Psychophysics, 1973, 14, 259-264.

Grant, D. A., Hake, H. W., Riopelle, A. J.. ¿ Kostlan, A. Effects of repeated pretesting with condit oned stimulus upon extinction of the conditioned eyelis response to light. American Journal of Psychology, 1951, 64, 247-251.

Grant, D. A., Hake, H. W., \& Schneider, D. E. Effects of pre-testing with the conditioned stimulus upon extinction of the conditioned eyelid response. American Journal of Psychology, 1948, 61, 243-246.

Lubow, R. E. Latent inhibition. Psychological Bulletin, 1973a, 79, 398-407

Lubow, R. E. Latent inhibition as a means of behavior prophylaxis. Psychological Reports, 1973b, 32, 1247-1252.

Milner, P. M. Physiological psychology. New York: Holt, Rinehart, \& Winston, 1970.

Perlmuter, L. C. Effect of CS manipulation on the conditioned eyelid response: compounding, generalization, the inter-CS interval, and preexposure. Psychonomic Science Monographs, 1966, 1, 271-286.

Schnur, P. Latent inhibition: The effects of nonreinforced preexposure of the CS in differential eyelid conditioning. Unpublished master's thesis, North Carolina State University, 1967.

Schnur, P., \& Ksir, C. J. Latent inhibition in human eyelid conditioning. Journal of Experimental Psychology, 1969, 80, 388-389.

Silver, A. Effects of prior CS presentations on classical conditioning of the skin conductance response. 
Psychophysiology, 1973, 10, 583588.

Sokolov, E. N., \& Paramanova, N. P. Concerning the role of the orientation reflex in the formation of motor conditioned reactions in man. Journal of Higher Nervous Activity, 1956, 6, 702-709. (Cited by E. N. Sokolov, Perception and the conditioned reflex. New York: Pergamon, 1963. Pp. 249).

Spence, $K$. W. Cognitive and drive factors in the extinction of the conditioned eyeblink in human subjects. Psychological Review, 1966, 73, 445-448.

Surwit, R. S. \& Poser, E. G. Latent inhibition in the conditioned electrodermal response. Journal of Comparative and Physiological Psychology, 1974, 86, 543-548.

Wolff, C., \& Maltzman, I. Conditioned orienting reflex and amount of preconditioning habituation. Proceedings of the American Psychological Association, 1968, 3, 129-130.
Zeiner, A. R. Orienting response and discrimination conditioning. Physiology and Behavior, 1970, 5, 641-646.

\section{NOTE}

1. This criterion was chosen because it was found in a pilot test that subjects who had not pressed to the CS after 31 presentations could not solve the problem even if the presentation was extended to $60 \mathrm{CSs}$.

(Received for publication October 11, 1974.) 